\title{
Resolução de problemas envolvendo soma e intersecção de subespaços vetoriais
}

\author{
José Carlos Pinto Leivas 1 \\ Universidade Franciscana (UFN), Programa de Pós-Graduação em Ensino de Ciências e \\ Matemática, Porto Alegre, RS, Brasil
}

\begin{abstract}
Resumo
Neste artigo, abordamos uma pesquisa qualitativa realizada no primeiro semestre de 2019 , com estudantes em ação continuada de uma disciplina envolvendo conteúdos de Geometria Analítica e Álgebra Linear. O estudo teve por objetivo desencadear os processos de visualização de soma e de intersecção de subespaços vetoriais, explorando aspectos geométricos por meio da Metodologia de Resolução de Problemas - MRP. Pretendemos, com isso, possibilitar aos participantes que atuam tanto na Escola Básica quanto no Ensino Superior abordagens metodológicas em que sejam efetivamente construtores do conhecimento. Foram propostas três atividades a serem desenvolvidas em sala de aula, seguindo passos indicados por autores consagrados sobre a MRP, assim como um problema sobre o mesmo tema, para ser resolvido fora da sala de aula e devolvido ao pesquisador. Os resultados mostraram que, inicialmente, os participantes não exploraram visualização nas etapas de resolução do primeiro problema, mas que a foram utilizando nas seguintes. Por fim, a etapa de avaliação, prevista como a última de cada um dos problemas, indicou que os indivíduos julgaram as atividades pertinentes no contexto de formação continuada em que estão inseridos, bem como que a metodologia proposta pode ser empregada, particularmente, se forem ensinar tal conteúdo.
\end{abstract}

Palavras-chave: resolução de problemas; soma e intersecção de subespaços vetoriais; ação continuada.

\section{Solving problems with sum and intersetion of vectorial espace}

\begin{abstract}
In this article, we approached a qualitative research carried out in the first semester of 2019, with students in continuous action of a discipline involving contents of Analytical Geometry and Linear Algebra. The purpose of this study was to develop the visualization processes of sum and intersection of vector subspaces, exploring geometric aspects through the Problem Solving Methodology - MRP. We intend, therefore to enable the participants who work both in the Basic School and in Higher Education methodological approaches in which they are effectively builders of knowledge. Three activities were proposed to be developed in the classroom, following steps indicated by wellestablished authors about MRP, as well as a problem on the same subject, to be solved outside the classroom and returned to the researcher. The results showed that, initially, participants did not explore visualization in the resolution steps of the first problem, but that they were used in the following steps. Finally, the evaluation stage, predicted to be the last of each of the problems,
\end{abstract}

${ }^{1}$ Doutor em Educação (Matemática) pela UFPR. Professor do Programa de Pós-Graduação em Ensino de Ciências e Matemática da Universidade Franciscana -UFN. Líder do Grupo de Estudos e Pesquisas em Geometria - GEPGEO. leivasjc@gmail.com.br. 
indicated that individuals judged the relevant activities in the context of continuing education in which they are inserted, and that the proposed methodology can be used, particularly if teach such content.

Keywords: problem solving; sum and intersection of vector subspaces; continued action..

\section{Resolución de problemas relacionados con la suma e intersección de subespacios vectoriales}

\section{Resumen}

En este artículo abordamos una investigación cualitativa realizada en el primer semestre de 2019, con estudiantes en acción continuada en una disciplina envolviendo contenidos de Geometría Analítica y Álgebra Lineal. El objetivo es desarrollar que el proceso de visualización de suma y intersección de subespacios vectoriales, explorando aspectos geométricos por medio de la Metodología de Resolución de Problemas de modo a posibilitar a los participantes que actúan tanto en la Escuela Básica como en la Enseñanza Superior, enfoques metodológicos en que sean efectivamente constructores del conocimiento. Se propusieron tres actividades a ser desarrolladas en el aula, siguiendo pasos indicados por autores consagrados sobre la MRP y un problema, sobre el mismo tema, para ser resuelto fuera del aula. Los resultados mostraron que, inicialmente, los participantes no exploraron la visualización en las etapas de resolución del primer problema, pero que la utilizaron en las siguientes. Por último, la evaluación, como última de las etapas, en cada uno de los problemas, indicó que los individuos juzgaron las actividades pertinentes en la formación continuada de la cual están participando y que la metodología propuesta puede ser empleada, particularmente, si son enseñar tal contenido.

Palabras clave: solución de problemas; suma e intersección de subespacios vectoriales; acción continua.

\section{Introdução}

O Álgebra Linear tem sido considerada como uma disciplina de carácter teórico de difícil compreensão pelos alunos dos diversos cursos que a têm incluída em suas grades curriculares. Em geral, o aspecto teórico envolvendo definições, teoremas, demonstrações conduz à dificuldade de percepção geométrica envolvida em problemas da área.

Em nosso entender, por nossa experiência no ensino superior, temos constatado que tal disciplina pouco ou nada tem contribuído com a Geometria Analítica, tornando os dois ramos da Matemática, de certo modo, separados, o que não faz sentido, uma vez que Álgebra Linear veio para dar um novo formato depois das mudanças oriundas de Descartes.

Lima e Gomes (2018) apresentam as inter-relações entre a Geometria Analítica e a Álgebra Linear na constituição desses dois campos. Para os autores, ao desenvolver e estabelecer o método analítico na resolução de problemas geométricos, ocorreram, entre os pesquisadores, duas críticas:

(i) A arbitrariedade aparentemente inútil de se utilizar um referencial, isto é, escolher um sistema de coordenadas que, apesar de fundamental para a resolução, não existia a priori; 
(ii) A introdução da linguagem algébrica ocasionava, em muitos casos, um excesso de cálculos e, consequentemente, com a utilização do formalismo algébrico, as etapas do raciocínio ou o senso geométrico acabavam sendo substituídos por uma grande quantidade de equações e notações (p. 11).

Para os autores, tais críticas levaram os estudiosos a desenvolver um cálculo geométrico que deu origem ao que é conhecido hoje como Álgebra Linear.

Courant e Robbins (2000) informam que, na aritmetização da Geometria, novos rumos foram dados “[...] já em 1629 por Fermat (1601-1655) e em 1637 por Descartes (1596-1650) em que a ideia fundamental da Geometria Analítica é a introdução de "coordenadas", isto é, de números vinculados ou coordenados com um objeto geométrico e caracterizando completamente este objeto" (p. 87).

Em Álgebra Linear, o elemento principal é denominado espaço vetorial, cujos elementos são chamados de vetores, os quais abstraem certos entes matemáticos, tornando-se necessário desenvolver visualização, a qual entendemos como "um construto mental, capaz de formar imagens mentais, com a finalidade de construir e comunicar determinado conceito matemático, com vistas a auxiliar na resolução de problemas analíticos ou geométricos” (LEIVAS, 2009, p. 22).

De acordo com Lima e Gomes (2018), conteúdos de Álgebra Linear foram introduzidos no Brasil na década de 1950, porém, como disciplina com essa denominação, somente em 1965. Essa cronologia é relativamente jovem e, talvez por isso, ainda inspire muitos cuidados e pesquisas que venham a fortalecer sua inclusão na formação de professores de Matemática, especialmente, buscando contribuir com a formação geométrica desses profissionais.

Com relação ao estado atual da Álgebra Linear na Licenciatura em Matemática, Prado e Bianchini (2018) fazem um estudo de sua inclusão em currículos de cinco regiões do país. Os autores buscaram averiguar como a disciplina é abordada, visando o professor que atuará na educação básica. Os autores concluem a pesquisa indicando ser "urgente a necessidade de reestruturação de disciplinas que abordem conceitos específicos de Matemática, pois é preciso estabelecer articulações entre as noções de Educação Matemática, noções de Matemática e noções matemáticas ensinadas na Educação Básica” (p. 88-89).

A partir dessas premissas, justifica-se a presente pesquisa, realizada na formação continuada de professores de Matemática, mais especificamente em disciplina de Fundamentos de Álgebra Linear e Geometria Analítica. O estudo teve por objetivo desencadear os processos de visualização de soma e de intersecção de subespaços vetoriais, explorando aspectos geométricos por meio da metodologia de Resolução de Problemas. Pretendemos, com isso, possibilitar aos participantes que atuam tanto na Escola Básica quanto no Ensino Superior, abordagens metodológicas em que sejam efetivamente construtores do conhecimento. 


\section{Revisão de Literatura}

Neste item, apresentaremos os fundamentos teóricos que nortearam a presente pesquisa, como segue.

\section{$\underline{\text { Resolução de Problemas }}$}

Embora a metodologia de Resolução de Problemas esteja consagrada mundialmente, no Brasil ela adquiriu um status ampliado, com relação às pesquisas de outros países, a partir dos trabalhos das pesquisadoras Lourdes de La Rosa Onuchic e Norma Suely Allevato, com a denominação de Metodologia de Ensino-Aprendizagem-Avaliação de Matemática através da Resolução de Problemas. Corroboramos com as pesquisadoras, particularmente, na presente pesquisa, ao empregá-la no ensino de um tema de Álgebra Linear, não trivial, que é a conceituação de "somas ${ }^{2}$ e intersecções" de subespaços vetoriais, focando na abordagem geométrica. Nesse sentido, acreditamos que, com essa abordagem ampliada em relação a outros autores, torna-se mais viável atingir os princípios emanados do $\mathrm{NCTM}^{3}$ : equidade, currículo, ensino, aprendizagem, avaliação e tecnologia (PRINCÍPIOS e NORMAS, 2008).

No que diz respeito ao emprego dessa metodologia na formação continuada de professores, Justilin e Noguti (2017) afirmam, em sua pesquisa, que "ela é trabalhada em $22 \%$ dos trabalhos analisados por meio de "encontros de formação" (p.49). Na mesma direção, na formação continuada de professores e de currículos em Portugal, Serrazina (2017) afirma que ela teve forte influência das publicações do NCTM, sendo uma capacidade transversal que todos os alunos devem desenvolver, tendo sido incluída em propostas dos programas de Matemática no país.

Allevato e Onuchic (2008), a respeito dessa metodologia, afirmam que, mesmo que o conteúdo ainda não tenha sido apresentado formalmente aos estudantes, os problemas podem ser aplicados para desenvolver uma disciplina, o que vai ao encontro da presente pesquisa. Ao trazer a Resolução de Problemas como estratégia de ensino, Van de Walle (2009) afirma: “A maioria, senão todos, dos conceitos e procedimentos matemáticos podem ser ensinados melhor através da Resolução de Problema" (p. 57), reafirmando as indicações das autoras Allevato e Onuchic (2008), previamente citadas.

Ao fazer referência a Hiebert et al. (1997), Van de Walle (2009) diz que, para este autor, "Um problema é definido aqui como qualquer tarefa ou atividade na qual os estudantes não tenham nenhum método ou regra já receitados ou memorizados e nem haja uma percepção por parte dos estudantes de que haja um método ‘correto' específico de solução” (p. 57, grifo do autor). O autor afirma, ainda, que ensinar por meio de Resolução de Problemas pode ser dificultoso, uma vez que requer

\footnotetext{
${ }^{2}$ Embora soma seja a nomenclatura para o resultado da Adição de números, ela é empregada como operação em A.L.

${ }^{3}$ National Council of Teachers of Mathematics - NCTM
} 
planejamento minucioso da atividade, de modo que esta favoreça a construção do conhecimento e não apenas a reprodução e a aplicação de fórmulas

Van de Walle (2009) aponta algumas razões para manter esforços no uso da Resolução de Problemas, a saber: concentrar a atenção dos alunos sobre as ideias e dar sentido às mesmas; desenvolver nos alunos a convicção de que eles são capazes de fazer Matemática e de que essa disciplina faz sentido; fornecer dados contínuos para a avaliação, os quais podem ser usados para tomar decisões educacionais; ajudar os alunos a ter bom desempenho e manter os pais informados; possibilitar um ponto de partida para uma ampla gama de alunos; envolver os estudantes, de modo que ocorram menos problemas de disciplina; desenvolver o potencial matemático.

Polya (2006) é um dos pioneiros na metodologia de Resolução de Problemas e, para ele, "Um dos mais importantes deveres do professor é o de auxiliar seus alunos, o que não é fácil, pois exige tempo, dedicação e princípios firmes" (p.1). Ao indicar quatro fases em sua teoria: (1) compreensão do problema; (2) estabelecimento de um plano; (3) execução do plano; e (4) retrospecto, o autor orienta o professor a escolher essa metodologia na organização do trabalho pedagógico.

Por sua vez, ao ampliar a metodologia, Onuchic e Allevato (1999) organizam as etapas da seguinte forma: proposição do problema; aluno é desafiado a utilizar conhecimentos prévios; discussão em pequenos grupos; professor incentivando e observando; alunos resolvendo em grupos; apresentando resolução; discussão em plenária; busca de consenso; professor formalizando o conteúdo; proposição de novos problemas.

A partir dessas considerações que fundamentam a metodologia, a adaptamos para aplicar em uma situação real específica, de modo a atender as exigências de formação dos participantes da pesquisa e da situação pedagógica apresentada.

\section{Visualização}

Visualização tem sido um tema de interesse em nossas pesquisas, a qual entendemos "como um processo de formar imagens mentais, com a finalidade de construir e comunicar determinado conceito matemático, com vistas a auxiliar na resolução de problemas analíticos ou geométricos" (LEIVAS, 2009, p. 22). Nessa direção, Fischbein (1987, p. 104) considera representações visuais como um importante fator de globalização, e afirma que “[...] Uma imagem visual não somente organiza os dados em estruturas significativas, mas é também um fator importante para orientar o desenvolvimento de uma solução analítica; representações visuais são essenciais dispositivos antecipatórios". Assim, na medida em que alunos de uma disciplina de Álgebra Linear mobilizam habilidades visuais para resolver problemas envolvendo soma e intersecção de espaços vetoriais, parece ser um elemento fundamental na Metodologia de Resolução de Problemas - MRP. 
Nessa direção, para Arcavi (1999, p.217), "Visualização é a habilidade, o processo e o produto de criação, interpretação, uso e comentário sobre figuras, imagens, diagramas, em nossas mentes, em papel ou com ferramentas tecnológicas, com a finalidade de desenhar e comunicar informações, pensar sobre e desenvolver ideias não conhecidas e avançar na compreensão". Portanto, explorar habilidades visuais na MRP para interpretar e resolver os problemas sugeridos pelo investigador, cujos dados serão analisados por meio dos registros fornecidos pelos indivíduos participantes, bem como dos seus comentários feitos em papel, parece indicar um caminho satisfatório para a formação continuada de professores.

Uma busca envolvendo Resolução de Problemas juntamente com soma e intersecção de subespaços

Neste item, trazemos algumas considerações a respeito de trabalhos publicados em nível de teses e dissertações sobre 'soma e intersecção' de subespaços vetoriais uma vez que, dos cinco alunos matriculados, para dois deles, $\mathrm{C}$ e $\mathrm{D}$, não tendo participado da atividade por motivos justificados que os impediram, optamos por envolvê-los no tema da aula para que pudessem acompanhar o desenrolar do conteúdo programático nas aulas subsequentes.

Ao indivíduo $\mathrm{C}$ foi destinada a tarefa de buscar por teses, no portal da CAPES, que abordassem o tema em apreço. Este aluno localizou duas que se aproximaram do pretendido, embora não atentando ao ensino e aprendizagem. O descritor empregado foi "resolução de problemas com soma e interseção de subespaços vetoriais" e com os seguintes filtros: "tese", "ciências exatas e da terra" e "matemática". Foram selecionadas duas delas se aproximando do pretendido.

$\mathrm{Na}$ primeira pesquisa identificada, seu autor cita constantemente a existência de espaços entrelaçados, e a tese foca em intersecções de subespaços. Na segunda encontrada, o autor analisa a ordem de convergência dos processos aproximatórios e envolve relação com a derivada forte de Laplace-Bertrami. Para tal, explora, novamente, a intersecção de subespaços invariantes. Percebemos, pois, que a MRP não foi envolvida nos dois trabalhos de tese.

Para a estudante D foi dada tarefa similar, porem buscando em dissertações no mesmo portal. Foi feita busca pelo descritor "resolução de problemas AND subespaços", não sendo localizada nenhuma relacionada ao ensino.

A partir desse rápido levantamento, encontramos mais uma justificativa para investigar a MRP em temas envolvendo Álgebra Linear.

\section{Metodologia}

\section{$\underline{\text { Tipo de pesquisa }}$}

Nossa pesquisa envolveu sujeitos em formação continuada, em uma disciplina de um Programa de Pós-Graduação em ensino. Em relação ao Ensino Superior, Severino (2016) afirma que, 
nesse nível, "O primeiro objetivo é o da formação de profissionais das diferentes áreas aplicadas, mediante o ensino/aprendizagem de habilidades e competências técnicas" (p.22), o que vai ao encontro dos nossos propósitos. Nesse processo, para a construção do objeto, torna-se essencial a pesquisa, inclusive para professores em formação que atuem ou pretendam atuar na Educação Básica, uma vez que, enquanto mestres e doutores, devem estar habilitados para isso. Para o autor, a pesquisa “[...] assume ainda uma dimensão pedagógica: a perspectiva decorrente de sua relação com a aprendizagem" (p.27) e isso vai ao encontro do que preconiza a MRP.

A presente pesquisa tem uma abordagem qualitativa, uma vez que não há tratamento estatístico de dados. Além disso, o estudo vem ao encontro do que sugere Severino (2016), para quem essa designação refere um conjunto de metodologias envolvendo, eventualmente, diversas referências epistemológicas, com o que concordamos, uma vez que a MRP pode focar em diversas tendências, como já explicitado anteriormente.

Em virtude de estarmos observando, por um lado, fenômenos e atitudes que emergiram da resolução de problemas, assim como, por outro lado, compartilhando conhecimentos e orientando os sujeitos no tempo de realização da pesquisa, defendemos, com base em Severino (2016), que o presente trabalho apresenta as características de uma pesquisa qualitativa participante. Amparados no mesmo autor, argumentamos, ainda, que ela pode ser tomada como de campo, uma vez que "o objeto/fonte é abordado em seu meio ambiente próprio. A coleta de dados é feita nas condições naturais em que os fenômenos ocorrem, sendo diretamente observados sem intervenção e manuseio por parte do pesquisador" (p. 131-132).

A respeito do tamanho do corpus da pesquisa, Bauer e Aarts (2015) dizem que "Devemos considerar o esforço envolvido na coleta de dados e na análise, o número de representações que se quer caracterizar, e alguns requisitos mínimos e máximos, por exemplo na análise automática do texto, como critérios para o tamanho do corpus" (p.59). Dessa forma, embora nossa pesquisa tenha envolvido apenas três estudantes de uma disciplina (diretamente, uma vez que outros dois também participaram de forma indireta), há de se considerar a situação em que ela ocorreu, isto é, em uma ação continuada, na qual o número de participantes é sempre muito reduzido.

No que diz respeito à análise dos dados coletados, evocamos a semiologia, pois ela "provê o analista com um conjunto de instrumentais conceptuais para uma abordagem sistemática dos sistemas de signos, a fim de descobrir como eles produzem sentido" (PENN, 2015, p. 319). Dessa forma, na busca de compreender como se realizam os registros dos indivíduos durante as etapas da MRP, consideramos, particularmente: os simbólicos, em língua natural e matemática; e os visuais, pelas representações geométricas. Além disso, ao avaliarem as atividades e resolverem novos problemas a 
posteriori da aula, é possível analisar tais registros à luz das escritas feitas em um tempo maior do que o utilizando durante a aplicação.

\section{Cenário da pesquisa}

A pesquisa foi realizada pelo pesquisador, em uma disciplina da qual é o professor, em um Programa de Pós-Graduação em Ensino de Ciências e Matemática, como parte do conteúdo programático dessa cadeira, durante o primeiro semestre letivo de 2019. Frequentaram a disciplina cinco estudantes, sendo dois de doutorado e três de mestrado. Como todos são oriundos de uma Licenciatura em Matemática, já a cursaram em algum momento na sua formação. No dia da aplicação, não se fizeram presentes um de doutorado e outro de mestrado, por motivo justificado.

$\mathrm{Na}$ aula em que a pesquisa foi desenvolvida, conforme o cronograma da disciplina, o tema a ser desencadeado era "soma e intersecção" de subespaços vetoriais. O investigador planejou desenvolvê-la por meio da MRP, em virtude de estar em um curso de ensino. Nesse sentido, vale reiterar o que foi indicado na justificativa deste artigo, especialmente no que diz respeito ao recomendado por Prado e Bianchini (2018), sobre incluir Álgebra Linear na formação do professor de Matemática, quer inicial ou continuada, ao que agregamos: explorando habilidades visuais a um tema até certo modo desenvolvido teoricamente.

Assim, planejamos a aula por meio de sete fichas que apresentavam desde o conhecimento de autores que abordam a MRP à resolução e à discussão de três problemas, inclusive com avaliações e sentimentos a respeito de sua resolução, ficando mais um problema para complementação após a aula, como é recomendado por Allevato e Onuchic (2008) em sua metodologia. Cada indivíduo escolheu um pseudônimo para evitar identificação nas respostas. São eles: $\boldsymbol{A}, \boldsymbol{B} . \boldsymbol{\pi}^{0}$. Os ausentes foram nomeados por $\mathrm{C}$ e $\mathrm{D}$.

Na sequência, passaremos à análise dos dados coletados, bem como os resultados alcançados.

\section{Resultados}

Com base nos fundamentos e etapas da MRP, adaptadas pelo investigador, iniciamos a coleta de dados da seguinte forma:

Ficha 1 - Você, certamente, já ouviu falar na Metodologia de Resolução de Problemas-MRP. Nesta aula, iremos explorá-la e, por isso, solicito que realizes e registres as atividades propostas na disciplina de Fundamentos de Álgebra Linear e Geometria Analítica-FALGA, inicialmente, de forma individual.

- Qual autor lhe vem à cabeça sobre a MRP em primeiro lugar?

- Recorda de outros? não ( ) $\operatorname{sim}($ ) qual ou quais?

As respostas à ficha 1 foram as seguintes: 
1. $\boldsymbol{A}$, Norma Allevato, enquanto $\boldsymbol{B}$ e $\boldsymbol{\pi}^{0}$ responderam Polya.

2. $\boldsymbol{B}$ e $\boldsymbol{\pi}^{0}$ disseram não lembrarem de outros autores. $\boldsymbol{A}$ registrou: Trabalhamos com outros autores na disciplina de Anos Iniciais, porém não recordo o nome. ${ }^{4}$

Ficha 2: A disciplina FALGA está abordando espaços e subespaços vetoriais, usualmente tratados na formação inicial de professores de Matemática.

-Você lembra o que é "soma de subespaços vetoriais"? (...) não (...)sim $\quad$ O que lembras:

$\boldsymbol{A}$ e $\boldsymbol{B}$ disseram não lembrarem do conceito.

$\pi^{0}$ disse lembrar-se e registrou: Sejam $U \subset V, W \subset V, U$ e $W$ subespaços do espaço vetorial $V$.

Definimos $U+W=\{\vec{u}+\vec{v} \mid \vec{u} \in U e \vec{v} \in W\}$.

-Você lembra o que é "intersecção de subespaços vetoriais"? (...) não (...)sim O que lembras:

$\boldsymbol{A}$ e $\boldsymbol{B}$ disseram não lembrarem dos conceitos.

$\pi^{0}$ registrou: Nas mesmas condições anteriores definimos $U \cap W=\{\vec{\imath} \mid \vec{\imath} \in U$ e $\vec{\imath} \in W\}$.

Ficha 3: A MRP é abordada por Onuchic e Allevatto, bem como outros autores: Polya, Hiebert, Kilpatrick, Van de Walle, os quais indicam etapas a serem seguidas. Para explorar tais etapas em problemas de FALGA, relembremos as definições a seguir:

Sejam S e U dois subespaços de um mesmo espaço vetorial V sobre um corpo K. Chamamos de soma dos subespaços o conjunto $\mathrm{W}$, cujos elementos são todos os vetores da forma $\mathrm{w}=\mathrm{s}+\mathrm{u}$, em que $s \in \mathrm{S}$ e $\mathrm{u} \in \mathrm{U}$.

Sejam S e W dois subespaços de um mesmo espaço vetorial V sobre um corpo K. O conjunto $\mathrm{S} \cap \mathrm{W}$ é também um subespaço vetorial e se denomina intersecção dos subespaços.

PROBLEMA PRELIMINAR- Em conjunto com seu colega do lado, a partir desse registro em linguagem natural das duas definições, faça um registro simbólico-algébrico para as duas.

$\boldsymbol{A}$ registrou na ficha fornecida pelo investigador:

\begin{tabular}{|l|l|}
\hline Soma de subespaços & $\mathrm{S}+\mathrm{U}=\left(\mathrm{s}_{1}, \mathrm{~s}_{2}, \mathrm{~s}_{3}, \ldots, \mathrm{s}_{\mathrm{n}}\right)+\left(\mathrm{u}_{1}, \mathrm{u}_{2}, \mathrm{u}_{3}, \ldots, \mathrm{u}_{\mathrm{n}}\right)=\mathrm{W}$ \\
\hline Intersecção de subespaços & $\mathrm{S} \cap \mathrm{W}=\left(\mathrm{s}_{1}, \mathrm{~s}_{2}, \mathrm{~s}_{3}, \ldots, \mathrm{s}_{\mathrm{n}}\right) \cap\left(\mathrm{w}_{1}, \mathrm{w}_{2}, \mathrm{w}_{3}, \ldots, \mathrm{w}_{\mathrm{n}}\right)=\mathrm{W}$ \\
\hline
\end{tabular}

Observamos, no registro feito pelo participante $\boldsymbol{A}$, ao que tudo indica, que não houve compreensão do problema de forma plena, uma vez que o mesmo não observou o conceito de soma e de interseção de subespaços vetoriais como sendo um conjunto (grifo nosso), usando notação inadequada. Lembramos que já estava sendo empregada tal notação em aulas anteriores à

${ }^{4}$ As transcrições são literais. 
investigação, quando o conceito de subespaço vetorial também havia sido trabalhado. De forma similar, o participante fez um registro na segunda linha para intersecção de subespaços, e foi além, ao denotar o resultado como sendo o próprio $\mathrm{W}$, o que pode ocorrer, por exemplo, quando o subespaço W for um subespaço do $\mathrm{S}$, sendo um caso particular e não generalizado, como no problema enunciado. O participante ainda assinala ao lado que os dois conjuntos resultantes são subespaços.

$\boldsymbol{B}$ registrou da seguinte forma:

\begin{tabular}{|l|l|}
\hline Soma de subespaços & $\mathrm{S}+\mathrm{U}=\mathrm{v}\left(\mathrm{v}_{1}, \mathrm{v}_{2}, \mathrm{v}_{3}, \ldots, \mathrm{v}_{\mathrm{n}}\right)+\mathrm{u}\left(\mathrm{u}_{1}, \mathrm{u}_{2}, \mathrm{u}_{3}, \ldots, \mathrm{u}_{\mathrm{n}}\right)$ \\
\hline Intersecção de subespaços & $\mathrm{S} \cap \mathrm{W}=\mathrm{v}\left(\mathrm{v}_{1}, \mathrm{v}_{2}, \mathrm{v}_{3}, \ldots, \mathrm{v}_{\mathrm{n}}\right) \cap \mathrm{u}\left(\mathrm{u}_{1}, \mathrm{u}_{2}, \mathrm{u}_{3}, \ldots, \mathrm{u}_{\mathrm{n}}\right)$ \\
\hline
\end{tabular}

O participante usou notação em nada formal e de acordo com o que seria apropriado para indivíduos que já cursaram a Licenciatura em Matemática, pelo menos, e cursam um programa de pós-graduação. Observamos que ele fez uma abreviação, realizando a operação de adição e a de intersecção entre os dois conjuntos, no primeiro membro da igualdade, enquanto que, no segundo, a operação é feita entre conjuntos, inclusive de forma incorreta na intersecção que é operação entre conjuntos e não entre dois vetores.

$\pi^{0}$ completou a tabela fornecida, explorando a linguagem matemática correta, como é desejado:

\begin{tabular}{|l|l|}
\hline Soma de subespaços & $\mathrm{S}+\mathrm{U}=\{\mathrm{s}+\mathrm{u} \mid \mathrm{s} \in \mathrm{S}$ e $\mathrm{u} \in \mathrm{U}\}$ \\
\hline Intersecção de subespaços & $\mathrm{S} \cap \mathrm{W}=\{\mathrm{i} \mid \mathrm{i} \in \mathrm{S}$ e $\mathrm{i} \in \mathrm{W}\}$ \\
\hline
\end{tabular}

A análise das três primeiras fichas de nossa investigação buscava averiguar o estágio em que os participantes se encontravam em termos de simbologia matemática, a qual já deveria ser de amplo conhecimento de egressos de uma Licenciatura em Matemática e participantes de uma disciplina em ação continuada em andamento, não sendo, portanto, algo novo, a menos dos conceitos que se apresentavam. Isso vem fortalecer o argumentado por Lima e Gomes (2018), quanto a críticas existentes entre pesquisadores da área, de que "A introdução da linguagem algébrica ocasionava, em muitos casos, um excesso de cálculos e, consequentemente, com a utilização do formalismo algébrico, as etapas do raciocínio ou o senso geométrico acabavam sendo substituídos por uma grande quantidade de equações e notações" (p. 11).

Em nosso entender, a linguagem matemática expressa em linguagem natural, como a do enunciado da questão investigativa, torna-se importante de ser convertida para a simbólica, a fim de que determinado conceito seja bem construído pelo aluno.

Na sequência da investigação, passamos a utilizar, propriamente, a MRP em um problema específico de Álgebra Linear com cunho analítico, esperando que os indivíduos pudessem explorar habilidades visuais na resolução deste. Até aqui foram indicadas as fichas, isto é, o que era encaminhado aos alunos para se situarem na metodologia iniciada e registrarem os primeiros passos 
da investigação. A partir da Ficha 4, com o enunciado do Problema 1, passamos a analisar etapa por etapa da MRP com as respectivas análises.

Ficha 4: Seja o espaço vetorial $\mathbf{R}^{2}$ e nele os subespaços:

- S é o conjunto dos pares ordenados de números reais cujas ordenadas são nulas;

- W é o conjunto dos pares ordenados de números reais cujas abscissas são nulas.

PROBLEMA 1 - Obter a soma e a intersecção dos dois subespaços.

$1^{\text {a }}$ etapa: leia o problema atentamente e responda: você entendeu o problema? Explique. Faça um registro simbólico/algébrico/geométrico dos conjuntos

A: Entendi, devo construir os dois conjuntos com as informações descritas e, após, realizar as operações.

$\mathrm{S}=\left\{\left(0, \mathrm{~s}_{1}\right),\left(0, \mathrm{~s}_{2}\right), \ldots,\left(0, \mathrm{~s}_{\mathrm{n}}\right)\right\} ; \mathrm{W}=\left\{\left(\mathrm{w}_{1}, 0\right),\left(\mathrm{w}_{2}, 0\right), \ldots,\left(\mathrm{w}_{\mathrm{n}}, 0\right)\right\}$.

$\mathrm{S}+\mathrm{W}=\mathrm{Y}$ novo conjunto e $\mathrm{S} \cap \mathrm{W}=X$ novo conjunto.

$\boldsymbol{B}$ : Entende-se que $S$ e $W$ são subespaços de $\boldsymbol{R}^{2}$, onde o conjunto $S$ tem pares ordenados reais onde $x$ é nulo e W tem pares ordenados reais cujos y são nulos e o problema quer a soma e a intersecção dos dois subespaços.

$\pi^{0}$ : Sim, entendi o problema. $\mathrm{S}=\left\{(\mathrm{x}, \mathrm{y}) \in \mathbf{R}^{2} \mid \mathrm{y}=\mathrm{o}\right\}$ e $\mathrm{W}=\left\{(\mathrm{x}, \mathrm{y}) \in \mathbf{R}^{2} \mid \mathrm{x}=\mathrm{o}\right\}$.

Ao desencadear a MRP, seguimos o indicado por Onuchic e Allevato (1999) no que diz respeito a etapa de proposição do problema, o qual foi escolhido para dar início ao tema da aula 'soma e interseção' de subespaços vetoriais. Nesse sentido, escolhemos um problema relativamente simples de ser compreendido, como de fato foi feito pelos três indivíduos. O problema envolvia a compreensão de coordenadas de pontos sobre os eixos cartesianos. Assim, partimos do conhecimento prévio desses participantes, de modo que pudessem ter confiança nas etapas que viriam a seguir.

Observamos que $\boldsymbol{A}$ realizou um registro simbólico dos dois conjuntos e indicou as duas operações a serem realizadas, enquanto $\boldsymbol{B}$ expressou apenas em linguagem natural sua compreensão do problema.

Por sua vez, $\pi^{0}$ registrou simbolicamente os dois conjuntos dados, também mostrando que compreendeu o problema, muito embora não concretizasse a simbologia das operações a serem realizadas.

Ficha 5: nesta ficha encaminhamos para a segunda etapa da MRP.

$2^{\mathbf{a}}$ etapa: elabore um plano prévio para a resolução do problema [sem partir para a resolução]

Esta ficha foi distribuída juntamente com a anterior, de modo que os indivíduos pudessem refletir um pouco mais sobre suas respostas por meio da comparação entre ambas. 
$A$ : foi bem sintética, registrando:

Construir os conjuntos;

Realizar as operações (soma e intersecção);

Registrar simbolicamente e formalmente os processos realizados.

$\boldsymbol{B}$ : criar os pares ordenados conforme indicado;

Obter a soma e a intersecção entre eles.

$\left.\pi^{0}: 1^{o}\right)$ Identificar os conjuntos $S$ e W.

$\left.2^{\circ}\right)$ Determinar $S+W$ e $S \cap W$.

Constatamos, nos registros dos três participantes que, depois de compreenderem o problema, elaboraram o seu plano de execução, o que corresponde à segunda etapa da MRP, fazendo isso de forma bastante simples e direta.

Ficha 6: nesta está encaminhada a terceira etapa da MRP.

$3^{\mathbf{a}}$ etapa: execute seu plano como você elaborou.

Uma vez que eram apenas três participantes e, afim de não prejudicar as etapas seguintes, onde há a discussão com seus pares, cada um deles, individualmente, registrou sua solução.

$\boldsymbol{A}$ : Este participante representou os subespaços por conjuntos como havia feito na $1^{\mathrm{a}}$ etapa, usando pares ordenados $S=\left\{\left(0, \mathrm{~s}_{1}\right),\left(0, \mathrm{~s}_{2}\right), \ldots,\left(0, \mathrm{~s}_{\mathrm{n}}\right)\right\} ; \mathrm{W}=\left\{\left(\mathrm{w}_{1}, 0\right),\left(\mathrm{w}_{2}, 0\right), \ldots,\left(\mathrm{w}_{\mathrm{n}}, 0\right)\right\}$, ou seja, seguiu o primeiro ponto de seu plano de resolução. Em seguida, seguiu o segundo ponto, efetuando a soma dos dois da mesma forma $\mathrm{S}+\mathrm{W}=\left\{\left(\left(0, \mathrm{~s}_{1}\right),\left(0, \mathrm{~s}_{2}\right), \ldots,\left(0, \mathrm{~s}_{\mathrm{n}}\right)+\left(\left(\mathrm{w}_{1}, 0\right),\left(\mathrm{w}_{2}, 0\right), \ldots,\left(\mathrm{w}_{\mathrm{n}}, 0\right)\right)\right\} \mathrm{e}\right.$, por fim, deu o conjunto resultante $\mathrm{S}+\mathrm{W}=\left\{\left(\mathrm{w}_{1}, \mathrm{~s}_{1}\right),\left(\mathrm{w}_{2}, \mathrm{~s}_{2}\right), \ldots,\left(\mathrm{w}_{\mathrm{n}}, \mathrm{S}_{\mathrm{n}}\right)\right\}$ indicando $\mathrm{S}+\mathrm{W}=\mathrm{Y} / \mathrm{y}=\mathrm{s}+\mathrm{w}$. Assim, cumpriu seu plano de resolução para a primeira operação, embora a simbologia inapropriada.

Imediatamente, realizou a segunda operação

$$
\mathrm{S} \cap \mathrm{W}=\left\{\left(\left(0, \mathrm{~s}_{1}\right),\left(0, \mathrm{~s}_{2}\right), \ldots,\left(0, \mathrm{~s}_{\mathrm{n}}\right)\right) \cap\left(\left(\mathrm{w}_{1}, 0\right),\left(\mathrm{w}_{2}, 0\right), \ldots,\left(\mathrm{w}_{\mathrm{n}}, 0\right)\right)\right\} .
$$

Aqui, tal estudante se equivocou na linguagem matemática, utilizando parênteses em vez de chaves para cada um dos componentes de cada conjunto, pois a intersecção é uma operação entre conjuntos. Isso, porém, não inviabilizou a sequência da resolução, conforme vemos no registro do estudante $\boldsymbol{A}$. $\mathrm{S} \cap \mathrm{W}=\{(0,0)\} \mathrm{e} \mathrm{S} \cap \mathrm{W}=\{\mathrm{X} / \mathrm{x} \in \mathrm{S}$ e $\mathrm{x} \in \mathrm{W}\}$, que mostra ter compreendido o problema e seguido seu planejamento para a segunda parte.

$\boldsymbol{B}$ : na compreensão do problema, dito participante expressou que os subespaços deveriam ser conjuntos constituídos por pares ordenados com características específicas. No seu plano, indicou criar os pares ordenados conforme indicado, o que executou, porém, equivocando-se nas notações empregadas, o que não inviabilizou a resolução.

$$
\mathrm{S}+\mathrm{W}=\left[\left(0, \mathrm{~s}_{1}\right)+\left(\left(\mathrm{w}_{1}, 0\right)=\left(\mathrm{w}_{1}, \mathrm{~s}_{1}\right)=\mathrm{w}+\mathrm{s}\right] \text { onde } \mathrm{x} \in \mathrm{S} \text { e } \mathrm{y} \in \mathrm{W}\right. \text {. }
$$


$\mathrm{S} \cap \mathrm{W}=\left[\left(0, \mathrm{~s}_{1}\right) \cap\left(\mathrm{w}_{1}, 0\right)=(0,0) \in(\mathrm{S} \cap \mathrm{W}\right.$, onde $\mathrm{x}$ S e $\mathrm{y} \in \mathrm{W}$.

$\pi^{0}$ : Havia entendido e representado perfeitamente o problema, elaborou seu plano e o executou. Retomou a notação inicial $S=\left\{(x, y) \in \mathbf{R}^{2} \mid y=0\right\}$ e $\mathrm{W}=\left\{(\mathrm{x}, \mathrm{y}) \in \mathbf{R}^{2} \mid \mathrm{x}=0\right\}$.

Seja $\mathrm{i} \in \mathrm{S} \cap \mathrm{W}$ e $\mathrm{i}=(\mathrm{x}, \mathrm{y})$. Como $\mathrm{i} \in \mathrm{S}$ vem que $\mathrm{y}=0$ e como $\mathrm{i} \in \mathrm{W}$, vem que $\mathrm{x}=0$. Portanto, $\mathrm{i}=(\mathrm{x}, \mathrm{y})$ $=(0,0)$ e, consequentemente, $\mathrm{S} \cap \mathrm{W}=\{(0,0)\}$. (Figura 1)

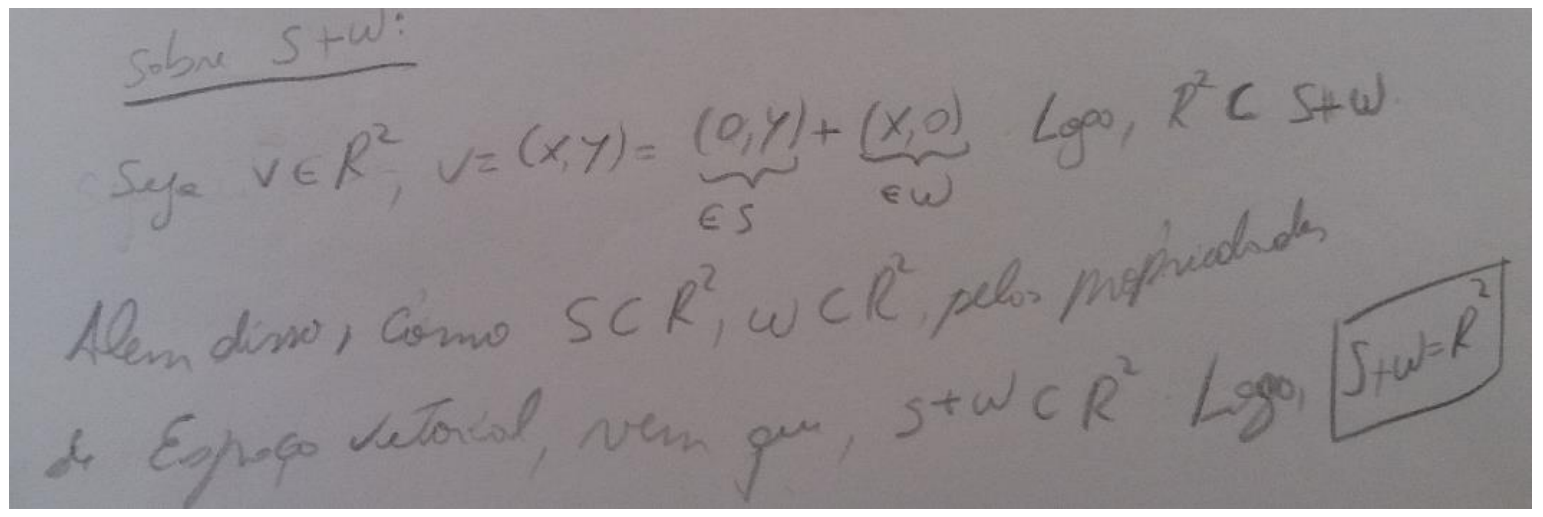

Figura 1: Registro de $\pi^{0}$ Fonte: próprio

Observamos, aqui, uma concordância com Severino (2016), quanto à natureza da pesquisa de campo em que os dados (no caso os registros escritos dos participantes) ocorreram de forma natural, sem consultas bibliográficas, apenas explorando os conhecimentos prévios dos estudantes, adquiridos na sua formação inicial ou na própria disciplina em curso. Assim, não houve preocupação maior do investigador quanto a diversidade de escrita matemática entre os três participantes na resolução do problema, até mesmo pela diferença de suas formações.

Percebemos, também, o indicado por Lima e Gomes (2018), quanto à constituição da Álgebra Linear no Brasil, em especial na formação do professor de Matemática. De acordo com esses autores, é necessário desenvolver e estabelecer o método analítico na resolução de problemas geométricos quando promovem críticas de pesquisadores sobre o cálculo algébrico. Nesse sentido, nenhum dos investigados utilizou representações geométricas para os subespaços vetoriais constantes do problema, haja vista que tem sido o foco da disciplina a exploração desse recurso visual. Ainda, parecem ser muito fortes os cálculos nas disciplinas do currículo da Licenciatura em Matemática.

Ficha 7: contém as orientações para a concretização da quarta etapa da MRP e, na sequência a quinta etapa.

$4^{\text {a }}$ etapa: faça um retrospecto de sua solução [revise-a]. Você mudaria algo? O quê?

Nessa etapa da MRP, os indivíduos foram solicitados a fazer uma retrospectiva da solução encontrada, ainda individualmente (POLYA, 2006). Todos eles indicaram que estavam de acordo 
com sua solução e que não mudariam nada. Há de observarmos que, mesmo os que não apresentaram rigor na escrita matemática, se deram conta de que poderiam aperfeiçoá-la.

$5^{a}$ etapa: discuta sua solução com seus pares expondo-a no quadro e dialogando com o professor.

Com as resoluções individuais, o investigador solicitou aos participantes da pesquisa apresentarem suas resoluções à frente do grupo. Cada um expôs seu pensamento, plano de execução e resolução. O investigador, então, dialogando com os participantes, levantou a hipótese de registros figurais dos subespaços do problema e os das soluções. Houve certo constrangimento de parte deles por não terem se dado conta das representações geométricas, isto é, tanto o subespaço $\mathrm{S}$, do eixo dos $\mathrm{X}$, quanto do $\mathrm{W}$, eixo dos $\mathrm{Y}$.

Foi retomada a importância de explorar habilidades visuais (ARCAVI, 1999) na resolução de muitos problemas, especialmente nos geométricos, como o da presente investigação, sobre o qual podem ser explorados comentários e imagens em diversos recursos didáticos.

Por sua vez, ao ampliar a metodologia, Allevato e Onuchic (2008) organizam as etapas da seguinte forma: proposição do problema; aluno é desafiado a utilizar conhecimentos prévios; discussão em pequenos grupos; professor incentivando e observando; alunos resolvendo em grupos; apresentando resolução; discussão em plenária; busca de consenso; professor formalizando o conteúdo; proposição de novos problemas.

O emprego da MRP para esta atividade foi relevante, o que reforça, especialmente, o preconizado por Van de Walle (2009) e Onuchic e Allevato (1999), de empregá-la como metodologia de ensino para diversas áreas do conhecimento.

Ficha 8: nela está o encaminhamento da sexta etapa, isto é, a avaliação da atividade realizada, segundo a MRP.

$6^{\mathbf{a}}$ etapa: faça uma avaliação sobre a atividade realizada

Onuchic e Allevato, ao denominarem Metodologia de Ensino-Aprendizagem-Avaliação de Matemática através da Resolução de Problemas, a justificam pela importância da avaliação no ensino de Matemática. Em virtude disso, solicitamos aos indivíduos registrarem seu sentimento com relação à atividade realizada, especialmente por serem professores e entendermos ser importante exercitarem o processo avaliativo, nesse momento todos eles ocupando a posição de estudantes. Seguem os registros feitos. (Figuras 2, 3 e 4) 


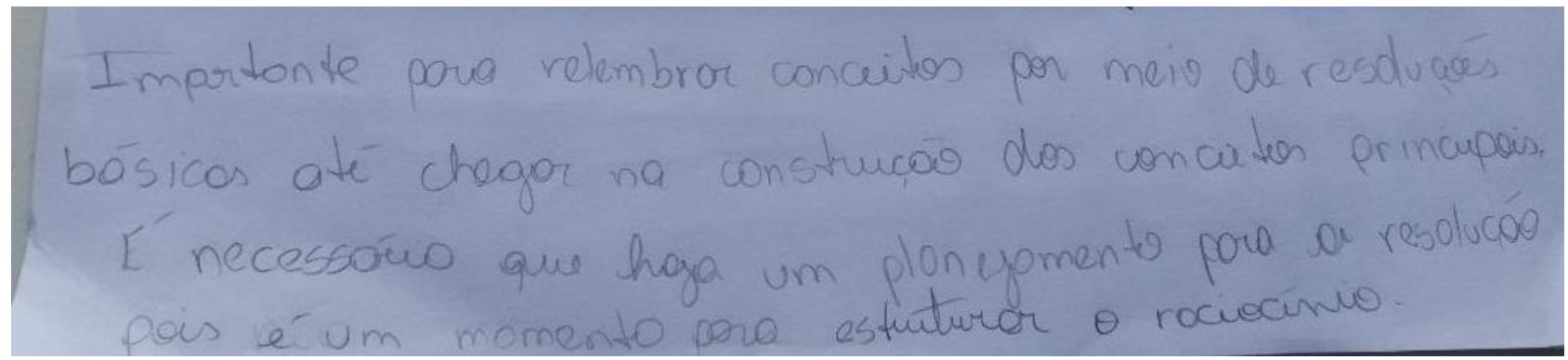

Figura 2: Registro de $A$.

Fonte: próprio

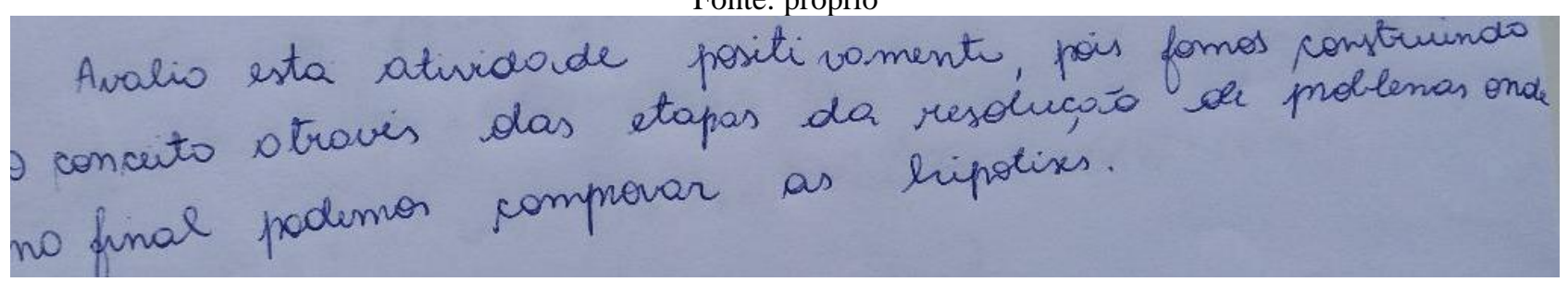

Figura 3: Registro de $B$.

Fonte: próprio

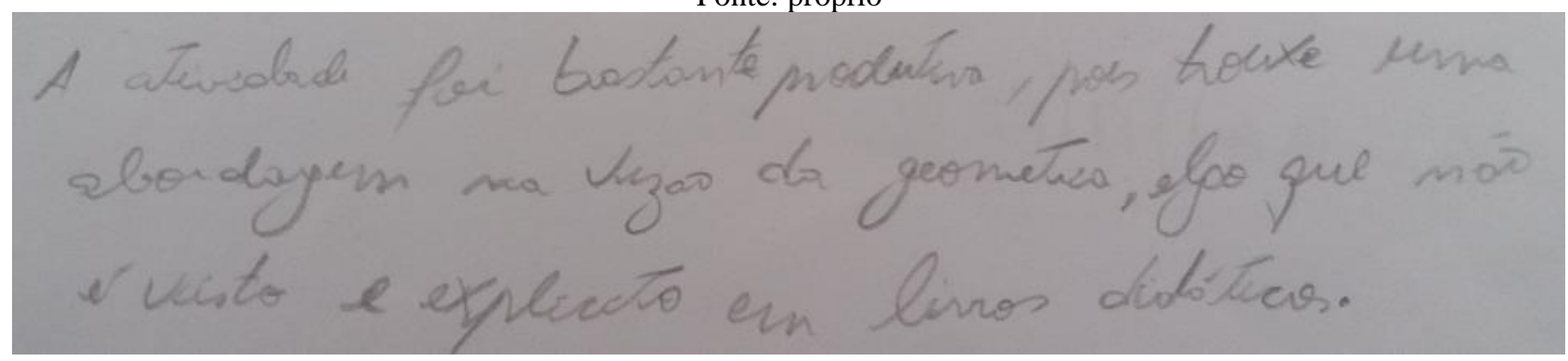

Figura 4: Registro de $\pi^{0}$.

Fonte: próprio

Dando continuidade às atividades envolvendo 'soma e intersecção' de subespaços vetoriais com a MRP, foi proposto o seguinte: repita a atividade realizada anteriormente, agora podendo ser feita em grupos para resolvê-la:

\section{PROBLEMA 2- Seja o espaço vetorial $\mathbf{R}^{3}$ e nele os subespaços:}

- U é o plano XOY; - W é o eixo OZ.

\section{- Obter a soma e a interseccão dos dois subespacos.}

Dessa feita, a metodologia foi empregada de forma mais dinâmica e, embora tivessem a possibilidade de realizar a atividade em duplas, os estudantes optaram por realizá-la individualmente, com pequenos comentários entre eles e o investigador. Percebemos, também, que a etapa da discussão e formalização no quadro, relativa à atividade 1, proporcionou uma melhoria na escrita de $\boldsymbol{A}$ e $\boldsymbol{B}$, particularmente. Além disso, $\boldsymbol{A}$ e $\pi^{0}$ utilizaram representação gráfica nas resoluções, como pode ser observado a seguir. 
eISSN: 2526-9062

$\boldsymbol{A}$ : Seguiu as etapas indicadas, como no problema anterior (Figura 5). Ao executar seu plano, obteve, algebricamente, a soma dos subespaços vetoriais e, para efetuar a intersecção, explorou a representação geométrica dos dois subespaços como sendo os eixos coordenados e a intersecção produzindo a origem do sistema cartesiano.

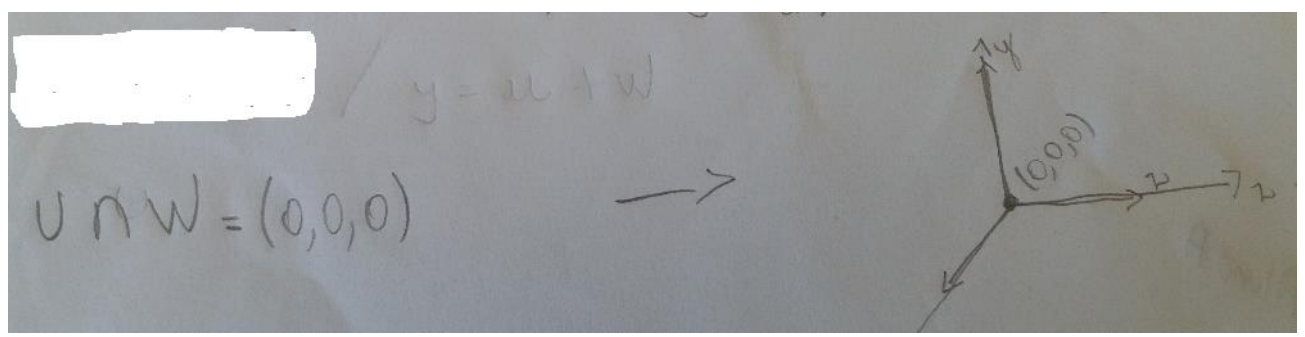

Figura 5: Registro de $A$.

Fonte: próprio

$\pi^{0}$ : Seu registro indicou: Resolução com apelo geométrico, mostrando que a habilidade visual, como indicada por Leivas (2009), parecendo ter sido despertada no indivíduo após a etapa da socialização da MRP recomendada.

Indo além, esse estudante afirmou: Retrospectiva: a solução mostrou-se adequada para a questão proposta.

Por fim, o indivíduo, seguindo a orientação dada na resolução daquele problema, fez sua avaliação deste: Utilizei a resolução geométrica, pois neste problema ela se apresenta como uma técnica a qual mostra-se eficaz na resolução da questão. (Figura 6)

Percebemos, assim, que a inclusão da avaliação na MRP, sugerida pelas educadoras Allevato e Onuchic (2008), são fundamentais, especialmente, na formação continuada de professores e no ensino de Álgebra Linear, uma vez que habilidades visuais se constituem ferramentas importantes para a compreensão de conceitos matemáticos diversos, sobretudo com relação ao trabalhado nesta investigação.

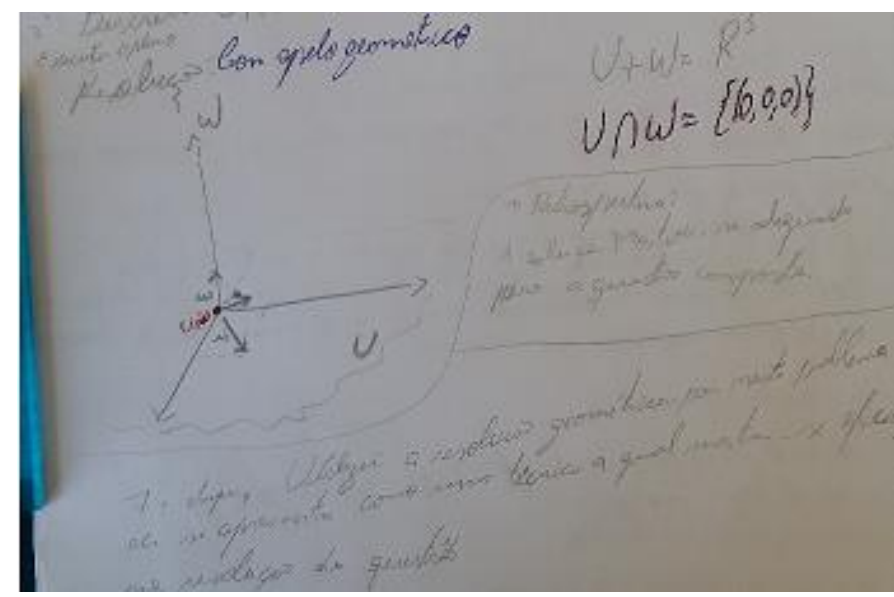

Figura 6: Registro de $\pi^{0}$. Fonte: próprio 
Considerando as resoluções deste problema, concluímos que está havendo um ganho no desenvolvimento do conteúdo em apreço, constante da programação da disciplina para ser realizada em 4 horas-aula, com a utilização da MRP, em relação a outros semestres letivos, em que o mesmo conteúdo foi desenvolvido com indivíduos nas mesmas situações de ensino.

Assim, reafirmamos o dito por Van de Walle (2009), de que "A maioria, senão todos, dos conceitos e procedimentos matemáticos podem ser ensinados melhor através da Resolução de Problema" (p.57). Também, confirmamos o dito por esse autor, de que "Um problema é definido aqui como qualquer tarefa ou atividade na qual os estudantes não tenham nenhum método ou regra já receitados ou memorizados e nem haja uma percepção por parte dos estudantes de que haja um método 'correto' específico de solução" (p.57, grifo do autor). Esse foi o caso na presente pesquisa, conforme levantamento inicial, de que os estudantes não lembravam ou não conheciam o conteúdo ou os autores relacionados a MRP. Assim, o segundo problema vai na direção das razões apontadas pelo autor, de que essa metodologia concentra a atenção dos alunos sobre as ideias, dando sentido às mesmas; desenvolvendo nos alunos a convicção de que eles são capazes de fazer Matemática e fornecendo dados contínuos para a avaliação.

A partir desse momento, o investigador, na busca de compreender melhor e avaliar o processo, propôs:

PROBLEMA 3- Seja o espaço vetorial $\mathbf{R}^{3}$ e nele os subespaços:

$$
\text { - } \mathbf{U}=\{(\mathrm{x}, \mathrm{y}, 0): \mathrm{x}, \mathrm{y} \in \mathbf{R}\} ; \quad-\mathbf{V}=\{(2 \mathrm{x}, 3 \mathrm{y}, 0), \mathrm{x}, \mathrm{y} \in \mathbf{R}\}
$$

\section{- Obter a soma e a interseccão dos dois subespacos}

A análise dos registros dos indivíduos nos permitiu concluir que todos eles exploraram representação geométrica nas resoluções. Isso vai ao encontro do que temos preconizado a respeito de explorar visualização como construto mental que tende a facilitar o processo de resolução de problemas, tanto geométricos quanto algébricos. No presente caso, estamos abordando um tema de Álgebra Linear, considerado difícil e teórico, com forte conotação algébrica e, até certo ponto, introduzidos recentemente no Brasil, conforme Lima e Gomes (2018). A análise corrobora, também, com Prado e Bianchini (2018), que afirmam ser "urgente a necessidade de reestruturação de disciplinas que abordem conceitos específicos de Matemática, pois é preciso estabelecer articulações entre as noções de Educação Matemática, noções de Matemática e noções matemáticas ensinadas na Educação Básica” (p.88-89).

Seguem as resoluções dos sujeitos. 
eISSN: 2526-9062

$\boldsymbol{A}$ : Este investigado expressou, algebricamente, os subespaços vetoriais, os representou no sistema cartesiano ortogonal $\mathbf{R}^{3}$ e forneceu o resultado das duas operações (Figura 7). Não chegou a representar o plano gerado pelos vetores resultantes, no entanto, mostrou estar começando a explorar os aspectos geométricos envolvidos.

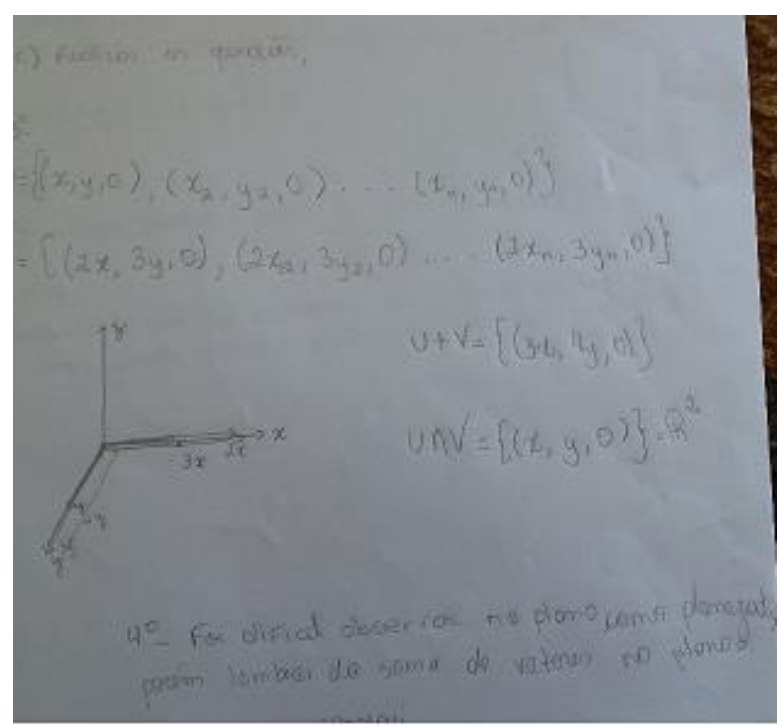

Figura 7: Registro de $A$.

Fonte: próprio

Registrou, ainda, que "Foi dificil observar no plano como planejado, porém lembrei da soma de valores no plano e consegui”. Quanto à avaliação da atividade, assim se expressou: Nesta atividade tive dificuldade de expressar as ideias de forma algébrica, logo, optei por fazer geometricamente e consegui encontrar o resultado.

Percebemos, nessa avaliação, a importância da visualização em Matemática, como indicado por Leivas (2009) "como auxiliar na resolução de problemas analíticos ou geométricos” (p. 22), e também Arcavi (1999, p. 217), a respeito de "pensar sobre e desenvolver ideias não conhecidas e avançar na compreensão".

$\boldsymbol{B}$ : Este participante, embora faça os registros seguindo etapas, se confundiu e se questionou na terceira etapa se a soma dos dois subespaços poderia produzir o $\mathbf{R}^{3}$. Expressou, na quarta etapa: Não consegui executar o plano na parte geometricamente e nem algebricamente.

Ele foi para a sétima etapa ao avaliar a atividade: Positivamente, pois fez questionar se poderia só admitir se era o $\boldsymbol{R}^{3}$. Porém pude perceber que não. (Figura 8) 
eISSN: $2526-9062$

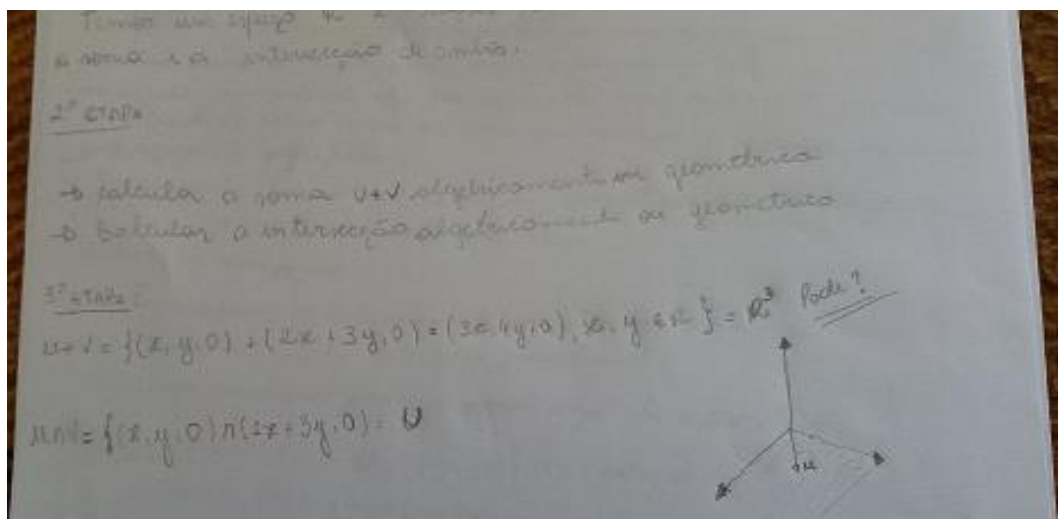

Figura 8: Registro de $B$.

Fonte: própria

$\pi^{0}$ : Registrou sua solução e, ao avaliá-la, indicou "Com esta questão eu consegui unir o enfoque algébrico e geométrico". (Figura 9)

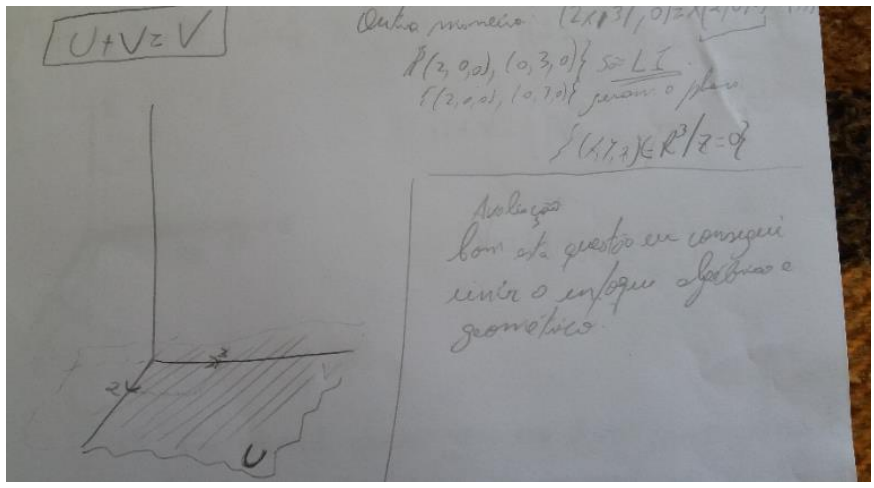

Figura 9: Registro de $\pi^{0}$.

Fonte: própria

Dessa forma, o objetivo da pesquisa já parece estar sendo atingido, uma vez que pretendíamos explorar a Geometria envolvida no tema da aula.

Como última fase dessa pesquisa, seguimos o indicado por Onuchic e Allevato (1999), de que o processo na MRP deve ter uma sequência com o professor propondo novos problemas aos alunos, os quais estejam diretamente relacionados ao problema que gerou o trabalho. Dessa forma, entendem as autoras que a aprendizagem é construída e o ensino reforçado. Assim, foi deixado para os alunos um novo problema a ser resolvido e encaminhado ao professor num prazo de duas semanas, a contar da aula em que o estudo foi realizado. Não nos ateremos a analisar as resoluções, uma vez que isso tornaria o artigo demasiado longo.

\section{PROBLEMA 4- Seja o espaço vetorial $\mathbf{R}^{3}$ e nele os subespaços:}

$$
\left.-\mathbf{U}_{\mathbf{1}}=\{(\mathrm{x}, \mathrm{y}, 0): \mathrm{x}, \mathrm{y} \in \mathbf{R}\} ; \quad-\mathbf{U}_{\mathbf{2}}=\{0, \mathrm{y}, \mathrm{z}): \mathrm{y}, \mathrm{z} \in \mathbf{R}\right\}
$$

- Obter a soma e a interseccão dos dois subespacos

$\boldsymbol{A}$ : intercalou a resolução algébrica com a geométrica e sinalizou: o plano foi contemplado, nas formas algébrica e geométrica. 
eISSN: $2526-9062$

B: informou: Não houve mudança do plano inicial pois executei o plano algebricamente.

$\pi^{0}$ : destacou o registrado na primeira etapa da MRP, a compreensão do problema feita por tal estudante. (Figura 10)

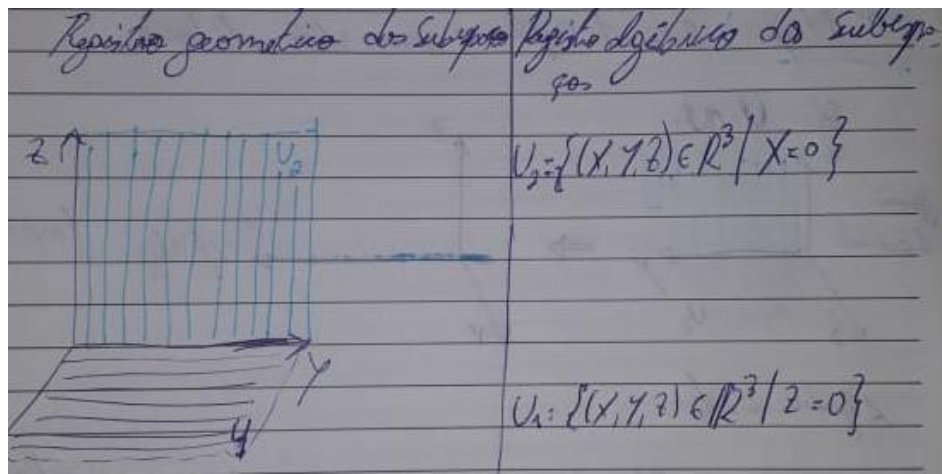

Figura 10: Registro de $\pi^{0}$.

Fonte: próprio

Ou seja, ele compreendeu os subespaços, tanto na forma geométrica quanto algébrica (Figura

11). Posteriormente, ao executar seu plano, assim o fez para a intersecção:

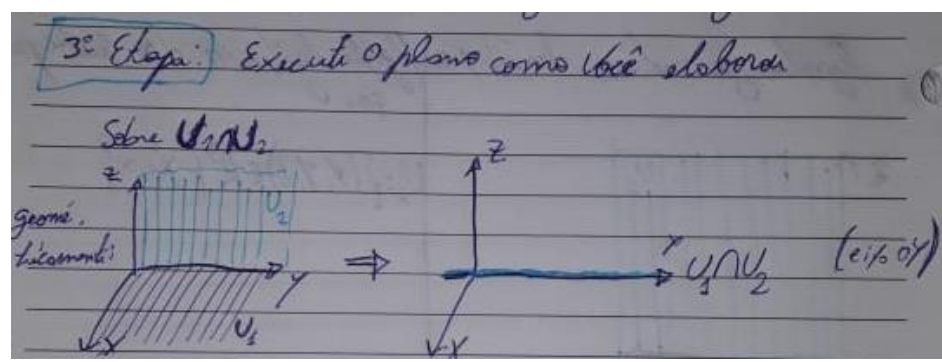

Figura 11: Registro de $\pi^{0}$.

Fonte: própria

Fez da seguinte forma para a soma (Figura 12):

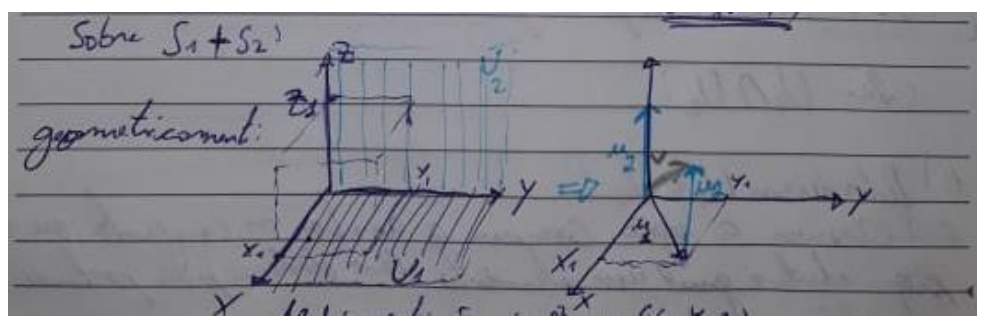

Figura 12: Registro de $\pi^{0}$.

Fonte: própria

O participante apresentou, também, os registros algébricos das duas operações.

No que segue, teceremos nossas considerações finais a respeito da investigação.

\section{Considerações finais}

Analisamos, neste artigo, uma investigação realizada com estudantes em ação continuada, explorando a MRP em uma situação real de sala de aula, na qual tivemos por objetivo desencadear processo de visualização de 'soma e intersecção' de subespaços vetoriais, explorando aspectos 
geométricos por meio da metodologia de Resolução de Problemas, de modo a possibilitar aos participantes, que atuam tanto na Escola Básica quanto no Ensino Superior, abordagens metodológicas em que sejam efetivamente construtores do conhecimento.

Para tal, fomos analisar os registros dos participantes na resolução, em sala de aula, de três problemas envolvendo 'soma e intersecção' de subespaços vetoriais, seguindo etapas sugeridas por estudiosos da MRP. Como ponto fundamental, verificamos que, no primeiro problema, os indivíduos não utilizaram representações geométricas na resolução, mas, a partir do debate na etapa final da metodologia, ponderaram a possibilidade de aplicar habilidades visuais nas resoluções. Essa discussão pareceu ter uma repercussão positiva na realização das demais atividades, uma vez que, instantes depois, os estudantes buscaram recorrer às habilidades visuais na resolução do problema 2 (parcialmente) e do problema 3 (quase integralmente).

As avaliações a respeito das tarefas propostas tiveram papel preponderante no desenrolar das resoluções e, particularmente, no último feito após a aula, encaminhado oportunamente ao investigador, atendendo ao recomendado por Allevato e Onuchic (2008) em sua metodologia. Dessa forma, percebemos que mobilizar habilidades visuais para resolver problemas envolvendo 'soma e intersecção’ de espaços vetoriais parece ser um elemento fundamental na MRP.

A pesquisa corrobora, ainda, com o indicado por Allevato e Onuchic (2008), de que, embora nenhum tema tenha sido apresentado formalmente aos estudantes os problemas propostos $\mathrm{e}$ resolvidos, juntamente com a metodologia empregada, podem servir para desenvolver uma disciplina.

\section{Referências}

ALLEVATO, N. S., ONUCHIC, L. R. Teaching mathematics in the classroom through problem solving. In: Research and Development in Problem Solving in Mathematics Education. ICME, México, 2008.

ARCAVI, A. The role of visual representation in the learning of mathematics. In: North American Chapter of The PME, 1999. Proceedings... Disponível em: <http://www.clab.edc.uoc.gr/aestit/4th/PDF/26.pdf>. Acesso em: 30 set. 2008.

BAUER, M.W.; AARTS, B. A construção do corpus: um princípio para a coleta de dados qualitativos. Em: BAUER, M. W.; GASKELL, G. Pesquisa Qualitativa com Texto, Imagem e Som: um manual prático. 13. Ed. Petrópois, RJ: Vozes, 2015.

COURANT, R.; ROBBINS, H. O que é Matemática? Rio de Janeiro: Editora Ciência Moderna Ltda, 2000.

FISCHBEIN, E. Intuition in science and mathematics: an educational approach. Dordrecht: Reidel, 1987. 
JUSTILIN, A. M. Formação de Professores e Resolução de Problemas: um Estudo a partir de Teses e Dissertações Brasileiras. In.: ONUCHIC, L. L. R; LEAL JUNIOR, L. C; PIRONEL, M. Perspectivas para resolução de problemas. São Paulo: Editora Livraria da Física, 2017.

LEIVAS, J. C. P. Imaginação, intuição e visualização: a riqueza de possibilidades da abordagem geométrica no currículo de cursos de licenciatura em Matemática. Tese (Doutorado em EducaçãoMatemática). Universidade Federal do Paraná. Curitiba, 2009, 294p.

LIMA, G. L.; GOMES, E. A Álgebra Linear: de sua construção como área de conhecimento matemático à sua inserção no currículo da primeira universidade brasileira. In: BIANCHINI, B. L. e MACHADO, S.D.A. (org.). Álgebra Linear sob o ponto de vista da Educação Matemática. São Paulo: Editora Livraria da Física, 2018.

ONUCHIC, L. R. ALLEVATO, N. S. G. Ensino-aprendizagem de Matemática através da Resolução de Problemas. In: BICUDO, M. A. V. (Org.) Pesquisa em Educação Matemática: concepções e perspectivas. São Paulo: UNESP, 1999. p. 199-218.

PENN, G. Análise Semiótica de Imagens Paradas. Em: BAUER, M. W.; GASKELL, G. Pesquisa Qualitativa com Texto, Imagem e Som: um manual prático. 13. Ed. Petrópolis, RJ: Vozes, 2015.

POLYA, G. A arte de resolver problemas. Rio de Janeiro: Interciência, 2006.

PRADO, E. A.; BIANCHINI, B. L. A Álgebra Linear na Licenciatura em Matemática. In: BIANCHINI, B. L. e MACHADO, S.D.A. (org.). Álgebra Linear sob o ponto de vista da Educação Matemática. São Paulo: Editora Livraria da Física, 2018.

\section{PRINCÍPOIS E NORMAS para a Matemática Escolar. National Council of Teachers of} Mathematis. 2. ed. Lisboa: Gabinete da APM, 2008.

SERRAZINA, L. Resolução de Problemas e Formação de Professores: um olhar sobre a situação em Portugal. In.: ONUCHIC, L. L. R; LEAL JUNIOR, L. C; PIRONEL, M. Perspectivas para resolução de problemas. São Paulo: Editora Livraria da Física, 2017.

SEVERINO, A.J. Metodologia do Trabalho Científico. 24. ed. ver. e atual. São Paulo: Cortez, 2016.

VAN de WALLE, J. A. Matemática no ensino fundamental: formação de professores em sala de aula. 6. Ed. Porto Alegre: Artmed, 2009. 